\title{
Investigaciones sobre gráficos estadísticos en Educación Primaria: revisión de la literatura
}

\author{
Pedro Arteaga \\ parteaga@ugr.es \\ Universidad de Granada, \\ España.
}

\author{
Danilo Díaz-Levicoy \\ dddiaz01@hotmail.com \\ Universidad de Granada, \\ España.
}

\author{
Carmen Batanero \\ batanero@ugr.es \\ Universidad de Granada, \\ España.
}

Recibido: Setiembre 20, 2016

Aceptado: Marzo 17, 2017

Resumen. En este trabajo se presentan resultados de una revisión de la literatura sobre los gráficos estadísticos con estudiantes de Educación Primaria. Para ello, se resumen investigaciones relacionadas a la lectura y construcción de estas representaciones, estableciendo similitudes entre los estudios. Esto nos permite generar marco de referencias para futuros estudios sobre esta temática, evidenciando las dificultades de los estudiantes frente a la lectura y los errores asociados a la construcción de diferentes gráficos.

Palabras clave: Gráficos estadísticos, Educación Primaria, Revisión de la literatura.

Abstract. In this paper, results of a literature review on statistical graphs with primary school students are presented. For this purpose, investigations that are related to reading and construction of such representations, establishing similarities between studies, are summarized below. This allows us to generate frameworks for future researches about this subject; highlighting students' difficulties at reading and errors associated with the construction of different graphics.

KeyWords: Statistical graphs, Primary Education, Literature review.

\subsection{Introducción}

La alta cantidad de información estadística que se trasmite por diferentes medios, exige que los ciudadanos tengan la capacidad para leerla e interpretarla, además de poder comunicar ideas en forma 
clara y precisa sobre el tema en cuestión. Una parte importante de esta información se transmite por medio de gráficos estadísticos (barras, líneas, sectores, pictogramas, entre otros), que son considerados elementos de la cultura estadística (Gal, 2002[18]) y, por consecuencia, del sentido estadístico (Batanero, Díaz, Contreras y Roa, 2013[5]). Autores, como Del Pino y Estrella (2012[11]) conciben la cultura estadística como un derecho ciudadano, implicando diferentes tareas, como:

(...) leer e interpretar los datos; usar argumentos estadísticos para dar evidencias sobre la validez de alguna afirmación; pensar críticamente sobre las afirmaciones, las encuestas y los estudios estadísticos que aparecen en los medios de comunicación; leer e interpretar tablas, gráficos y medidas de resumen que aparecen en los medios; interpretar, evaluar críticamente y comunicar información estadística; comprender y utilizar el lenguaje y las herramientas básicas de la estadística; apreciar el valor de la estadística en la vida cotidiana, la vida cívica y la vida profesional en calidad de consumidor de datos, de modo de actuar como un ciudadano informado y crítico en la sociedad basada en la información (p. 55).

Por su parte, las directrices curriculares de diferentes países han incorporado los contenidos de estadística y probabilidad desde los primeros cursos de la formación obligatoria (e.g., MECD, 2014[26]; MINEDUC, 2012[25]), entre ellos los gráficos estadísticos, como una forma de entregar herramientas a los estudiantes para enfrentarse con éxito a estos temas en diferentes situaciones de la vida cotidiana (social, personal y profesional). Estos gráficos estadísticos muchas veces son manipulados para sacar provecho de una determinada situación; por tanto se espera que los ciudadanos estadísticamente cultos identifiquen estas situaciones y puedan formarse una opinión fundada al respecto, lo que implica el dominio de los elementos específicos de cada gráfico sepan interpretarlos. Más aún, en muchas ciencias, los gráficos estadísticos cumplen un papel fundamental para organizar los datos que permiten observar relaciones entre variables, útiles para detectar los patrones, propiedades y relaciones en determinados fenómenos (Glazer, 2011[19]).

Lo anterior, motiva el desarrollo de un estudio en el que se desea aproximarse al estado del arte respecto a las investigaciones sobre lectura y construcción sobre gráficos estadísticos en Educación Primaria.

\subsection{Los gráficos estadísticos y sus elementos}

La comprensión de un gráfico estadístico se puede entender como "las habilidades de los lectores de gráficos para interpretar el significado de gráficos creados por otros o por ellos mismos" (Friel, Curcio y Bright, 2001, p. 132[17]). Esta visión supone entender la función y utilidad de cada elemento que constituye el gráfico estadístico con el que se esté trabajando. Es así, como diferentes autores han analizado y caracterizado los elementos, tal como lo describimos a continuación.

Kosslyn (1985[23]) menciona que para trabajar con gráficos estadísticos nos debemos fijar en los siguientes elementos:

Investigaciones sobre gráficos estadísticos en Educación Primaria: revisión de la literatura. D. Díaz-Levicoy et al Derechos Reservados ( 2017 Revista digital Matemática, Educación e Internet (http://tecdigital.tec.ac.cr/revistamatematica/) 
- El plano de fondo. Corresponde a la parte del gráfico estadístico que se utiliza como soporte del mismo; aunque con frecuencia es simplemente un fondo liso, en algunos gráficos se incluyen dibujos o fotografías que aluden a su contenido.

- Estructura del gráfico. Componentes que proporcionan información sobre lo que se está representado y la forma en que se representan. Dependiendo del gráfico, dicha estructura puede estar formada por un sistema de ejes cartesianos dimensional o tridimensional. Una estructura diferente aparece, por ejemplo, en los gráficos de sectores o el gráfico de cajas.

- Contenido pictórico. Son los elementos que se usan para representar los datos, por ejemplo los rectángulos en los histogramas, los puntos en los diagramas de puntos y dispersión, las líneas en los gráficos de líneas, polígono de frecuencias y ojivas, entre otros.

- Rótulos. Palabras y números que proporcionan información sobre las variables representadas y escalas de medida. Se colocan en el título del gráfico y los ejes.

Curcio (1987[9]) indica los siguientes elementos constituyentes de los gráficos estadísticos.

- Palabras o expresiones. Se refiere al texto que aparece en el texto, etiquetas en ejes y escalas, y permiten entender el contexto de la información proporcionada en el gráfico, así como las variables y la relación que se establece entre ellas.

- Contenido matemático que subyace en el gráfico estadístico. Ejemplo de ellos son los conjuntos numéricos que se utiliza, la longitud de las líneas en un gráfico de líneas o polígono de frecuencia, así como el área en el gráfico de sectores o pictograma, etc.

- Convenios específicos. Se refiere a aspectos propios de cada tipo de gráfico, y que son necesarios para abordar con éxito la lectura y construcción de cada gráfico, por ejemplo la proporcionalidad entre amplitud del sector circular y la frecuencia de cada categoría cuando se trabajo con un gráfico de sectores.

Más tarde Friel, Curcio y Bright (2001[17]) los reformulan en la forma siguiente:

- Título y etiquetas. Relacionado con el contenido contextual de la información mostrada en el gráfico, por ejemplo permite identificar las variables que se están representando.

- Marco del gráfico. Se relaciona con la información que se muestra en los ejes, escalas y marcas, con ellas se puede conocer, entre otras cosas, las magnitudes utilizadas y rango de valores que se consideran.

- Especificadores. Son elementos propios y específicos de cada gráfico estadístico, que son utilizados para la representación de la información. Como ejemplo por ejemplos las líneas en los gráficos de líneas y polígono de frecuencias.

- Fondo. Hace referencia los colores, cuadrículas e imágenes sobre la que se construye en gráfico estadístico.

Finalmente citamos a Alaminos (1993[1]) proporciona recomendaciones para la construcción de un gráfico, como las siguientes:

- Título y subtítulos que informa sobre el contenido del gráfico.

- Etiquetado de ejes. Deben estar marcadas las escalas en los ejes, así como las unidades de medida que se estén empleando, con suficientes divisiones. En los gráficos en que intervengan ejes cartesianos se debe indicar el cero cuando este forme parte de la escala. Igualmente se debe indicar el rango o valor máximo de los datos representados. En el caso que una parte del gráfico no es utilizado se debe señalar la discontinuidad, pero indicando siempre la base de referencia. 
- Otros elementos. Sugiere la necesidad de diferenciar con claridad las diferentes variables y categorías, usando colores o líneas de diferente tipo. También se debe indicar la fuente del gráfico y proporcionar la información complementaria necesitada para su interpretación, así como cuadriculado, si esto facilita la lectura. También comparan la forma en que se visualiza la información usando longitud, área y volumen, donde la forma más efectiva es la primera.

\subsection{Comprensión de gráficos estadísticos}

Bertin (1967[6]) entiende un gráfico estadístico como un sistema semiótico complejo, que necesita la compresión de cada uno de sus elementos por separado y una interpretación conjunta de los mismos. Es así, como este último autor plantea una teoría semiótica respecto al proceso de lectura de un gráfico, con los siguientes pasos.

- Identificación externa. Es la identificación de elementos conceptuales y de la vida cotidiana que están representados en el gráfico estadístico, los que se obtienen tras observar los rótulos alfanuméricos (título y etiquetas). Con ello se obtiene información sobre las variables representadas, el origen de los datos, el propósito del gráfico, tamaño de la muestra o población de datos, etc.

- Identificación interna. Es la valoración de la variabilidad del gráfico, estableciendo una relación entre los ejes cartesianos y las dimensiones visuales (escalas del gráfico), con las magnitudes y variables representadas en cada eje, el rango de variación de las mismas, las unidades de medidas y el factor de escala, si corresponde.

- Percepción de la correspondencia. Es el último paso, consiste en la puesta en relación o correspondencia de cada uno de los elementos del gráfico con la realidad representada. Con ello se obtienen conclusiones sobre las variables, su rango de variación, su distribución y sus características, así como sobre la relación entre las variables en la situación real representada.

Que una persona sepa establecer las relaciones anteriores en la interpretación o la construcción de gráficos significa que tiene una comprensión gráfica.

Wu (2004[32]), por su parte, menciona cuatro componentes para la comprensión de los gráficos estadísticos.

- Lectura gráfica. Corresponde a la capacidad de leer correctamente la información estadística contenida en un gráfico. Por ejemplo, obtener algún valor máximo o mínimo.

- Construcción gráfica. Consistiría en la construcción del gráfico estadístico de manera correcta a partir de datos obtenidos de diferentes fuentes (datos brutos, en tablas o otros gráficos).

- Interpretación gráfica. Va más allá de la lectura y consiste en interpretar la información del gráfico de acuerdo a su contexto.

- Evaluación de gráficos estadísticos. Está asociado a la calidad de la información mostrada y a la pertinencia del gráfico estadístico usado en la representación.

Otros autores han puesto su interés en estudiar las dificultades al abordar cuestiones sobre la información de un gráfico estadístico, ya que sobre una misma representación se pueden formular distintas

Investigaciones sobre gráficos estadísticos en Educación Primaria: revisión de la literatura. D. Díaz-Levicoy et al

Derechos Reservados @ 2017 Revista digital Matemática, Educación e Internet (http://tecdigital.tec.ac.cr/revistamatematica/) 
preguntas y relacionar diferentes elementos (objetos matemáticos).

En tal sentido, Wainer (1992[30]) clasifica en tres los tipos de preguntas que se pueden plantear a partir de un gráfico estadístico:

- Nivel elemental. Preguntas relacionadas únicamente con la extracción de datos directos del gráfico estadístico.

- Nivel intermedio. Preguntas relacionadas con la evaluación de tendencias basándose en una parte de los datos.

- Nivel avanzado. Preguntas acerca de la estructura profunda de los datos presentados en su totalidad, usualmente comparando tendencias y viendo agrupaciones.

Más tarde, Curcio (1989[10]) y Friel, Curcio y Bright (2001) definen los siguientes niveles:

- Leer los datos. Es la lectura literal de información del gráfico estadístico, es decir, no se realiza interpretación ni cálculos. Por ejemplo, determinar la frecuencia para una determinada categoría o bien la operación inversa, dada una frecuencia encontrar a qué categoría corresponde.

- Leer dentro de los datos. Es la lectura basada en los datos proporcionados en el gráfico, pero que no se presentan explícitamente; en este nivel se realizan cálculos o se buscan relaciones en función a la información mostrada en el gráfico estadístico. Un ejemplo es calcular la media aritmética con la información del gráfico.

- Leer más allá de los datos. Es la realización de inferencias o predicciones con la información del gráfico. Concretamente, consiste en predecir tendencias o valores, de acuerdo al contexto del la información. También puede consistir en interpolar o extrapolar un valor del gráfico.

Como ejemplo, podemos mencionar la estimación de los valores de una distribución con el paso del tiempo y que no están explicitados en el gráfico.

- Leer detrás de los datos. Es el nivel más alto y está asociado a una valoración crítica de los datos, del tipo de gráfico utilizado, de la manera en que se han obtenido y analizado los datos. Por ejemplo, analizar si la elección de la muestra considerada en el estudio es la adecuada; o si las conclusiones obtenidas son adecuadas a la información y el contexto representado.

Del mismo modo, autores han centrado su interés en la construcción de gráficos. Es así como Arteaga (2011[2]) y Batanero, Arteaga y Ruiz (2010[4]) proponen cuatro niveles asociados a la construcción de gráficos estadísticos, bajo la premisa que la construcción de gráficos estadísticos es una actividad semiótica que puede ser compleja según los objetivos matemáticos que intervienen. Estos autores proponen los siguientes niveles de complejidad semiótica.

- Representación de datos individuales. Se asocia a la representación de datos aislados (puede ser un dato o una porción de ellos), sin realizar una representación conjunta de ellos. En esta construcción no son utilizadas las ideas de variable y distribución, por lo que no se realiza un análisis global de la información. Esta situación ocurre cuando un estudiantes es capaz de graficar solo su edad, y quizás de algunos compañeros más de clase, frente a la tarea de construir un gráfico con las edades de toda la clase.

- Representación de un conjunto de datos sin llegar a resumir su distribución. Está asociado a la representación de cada dato, de la distribución sobre un gráfico, sin agruparlos y sin usar las ideas de frecuencia y distribución de frecuencias, aunque ya se maneja la idea de variable. Un estudiante alcanza este nivel si realiza un gráfico con 20 barras, donde cada barra representa la edad de cada compañero de clase.

- Representación de una distribución de datos. Se refiere a la representación de una distribución, agrupando los datos y calculando las frecuencias respectivas; los datos son mostrados en forma ordenada, en general, según los ejes cartesianos. Un estudiante alcanza este nivel cuando es 
capaz de agrupar las edades de una clase, calculando frecuencias y representen las edades en orden (dado por el eje X).

- Representación de varias distribuciones sobre un mismo gráfico. Se alcanza este nivel cuando se es capaz de representar de dos o más distribuciones de frecuencias en el mismo gráfico estadístico. Una situación que ejemplifica esto es cuando un estudiante puede graficar las edades de sus compañeros de clase separando por género.

Lo anterior permite confirmar la complejidad de las actividades asociadas a los gráficos estadísticos, aportando elementos que se pueden considerar en estudios futuros. En lo que sigue se exponen los resultados, sobre lectura y construcción, de algunas investigaciones con estudiantes de Educación Primaria, resultados que pueden aportar al estado del arte de los estudios en esta área.

\subsection{Investigaciones sobre lectura de gráficos estadísticos}

Investigaciones sobre la lectura de gráficos estadísticos son emergentes en la Educación Primaria, muchas de ellas motivadas por la inclusión de este temática en las directrices curriculares desde los primeros cursos y la necesidad de formar estudiantes estadísticamente cultos. A continuación, se describen los resultados de algunos de los estudios.

Guimarães (2002[21]) estudia la interpretación de los gráficos de barras por 107 estudiantes de $3^{\circ}$ de Educación Primaria en Brasil, plantando 3 actividades (dos con datos nominales y uno con datos ordinales). Los resultados muestran que el $72 \%$ del estudiantes logran la lectura puntual (encontrar máximos, mínimos y localizar frecuencias o categorías), presentado dificultades aquellas actividades en donde se debe leer una frecuencia que no está explicita. Se observan dificultades cuando se aborda una comprensión variacional, en las que se debe localizar la parte del gráfico en que la variable estudiada sufre su mayor aumento o disminución, que alcanza solo un $26,3 \%$ de respuestas correctas. Finalmente, el 54,2\% de los estudiantes responde con éxito la actividad en que deben realizar una extrapolación.

Pagan, Leite, Magina y Cazorla (2008[27]) estudian la lectura e interpretación de tablas y gráficos estadísticos de 399 estudiantes (159 de $5^{\circ}$ grado y 80 de $8^{\circ}$ grado de Educación Primaria y 160 de $2^{\circ}$ de Educación Secundaria) en Brasil. Dos de sus ítems están relacionados con gráficos. Sus resultados muestran un porcentaje de logro de $67,3 \%$; obteniendo mejores resultados en las actividades que demandan un nivel de lectura 1 ( $84 \%$ de respuestas correctas) frente al $43 \%$ en las actividades que se exige un nivel 2 de lectura, según los niveles descrito por Curcio.

Fernandes y Morais (2011[15]) estudia los niveles de lectura que alcanzan 108 estudiantes de $9^{\circ}$ grado de Educación Fundamental en Portugal. Para ello, se aplica un instrumento con tres actividades, en la primera se presenta un gráfico de barras, la segunda un diagrama de sectores y la tercera un diagrama de líneas dobles. Cada actividad tenía tres o cuatro preguntas que se corresponde a los niveles de lectura descritos por Curcio. Los resultados muestran que los estudiantes no presentan mayores dificultades para abordar las preguntas de nivel 1, el de lectura literal, no así en los niveles 2 y 3 . Las preguntas de nivel 2 presentan una diferencia levemente mayor que las de nivel 3, que los autores 
relacionan a la falta de conocimiento matemático y errores de interpretación. Las dificultades de las preguntas de nivel 1 se deben a que no se observó todos los elementos del gráfico o por una lectura errada del enunciado. En las preguntas de nivel tres se observa que los estudiantes no conocían el contexto, lo que impedía hacer predicciones o inferencias.

Carvalho, Campos y Monteiro (2011[7]) presentan los resultados de dos estudios relacionados con la inferencia (relación) directa e inversa en la interpretación de gráficos de líneas, por niños ingleses de 11 a 14 años de edad. La muestra de estudiantes estuvo constituida por 270 niños pertenecientes a cuatro escuelas públicas del Distrito de Oxfordshire (Inglaterra). En primer estudio consideró una muestra de 84 estudiantes de $7^{\circ}, 8^{\circ}$ y $9^{\circ}$ años de escolaridad de Oxford, se basa en actividades de inferencia directa e inversa. En el segundo estudio, con 186 estudiantes de los mismos niveles educaciones de Cowley, Kidlington y Holton, se abordan problemas de inferencia directa e inversa en gráficos de pendientes positivas y negativas. Los resultados muestran que los estudiantes responden con mayor éxito las actividades de inferencia directa, y que estos son mejores a medida que avanzan en los niveles de escolaridad. Como principal error está el considerar la situación en que interviene una inferencia inversa como directa, ya que sus respuestas se centran en aspectos visuales.

Evangelista (2013[13]) analiza el desempeño de una muestra de 60 niños de $5^{\circ}$ curso de Educación Primaria en Brasil al trabajar con gráficos de barras y de líneas. En la investigación se propone a los niños 8 actividades de lectura e interpretación de gráficos con actividades de nivel de lectura 1 y 2 según Curcio. Los resultados muestran que los niños contestan correctamente el $51 \%$ de las actividades planteadas. Los mejores resultados se alcanzan en los gráficos de barras y los de menor nivel de logro $(31 \%)$ en un diagrama de líneas dobles. En promedio, los estudiantes responden correctamente el $59 \%$ de las actividades relacionadas con gráficos de barras y el $43 \%$ a las de gráficos de líneas. Sobre el tipo de pregunta vemos que las actividades de localización de frecuencias o categorías tienen un nivel de logro de $60 \%$, seguido las de localizar la moda con un $51 \%$ y suma las de valores con un $41 \%$.

Cruz (2013[8]) estudia las dificultades al leer gráficos estadísticos, en una clase de 21 estudiantes de $3^{\circ}$ de Educación Primaria en Lisboa. La investigación contempla la aplicación de un pre test, una intervención de aula y un post test. Este incluye dos actividades relacionadas a la lectura de gráficos (un pictograma y un gráfico de sectores). Los resultados muestran que los estudiantes alcanzan mejores resultados al trabajar con el pictograma que con el gráfico de sectores, con un $70 \%$ y $21,3 \%$ de éxito, respectivamente. Respecto a los niveles de lectura de Curcio (1989[10]) los estudiantes, independiente del tipo de gráfico, responden correctamente el 53,8\% de las actividades de nivel 1; el 46,8\% de las del nivel 2; y $24,5 \%$ de las que se encuentran en un nivel 3. Además, se muestra que los estudiantes han mejorado sus resultados tras la intervención de aula.

\subsection{Investigaciones sobre construcción de gráficos estadísticos}

La construcción de gráficos no es una actividad sencilla, pese a que muchas veces se considere así. Esto se debe a que cada gráfico estadístico ocupa diferentes objetos matemáticos que intervienen en la construcción, por ejemplo no usamos los mismos conceptos matemáticos al construir un gráfico de

Investigaciones sobre gráficos estadísticos en Educación Primaria: revisión de la literatura. D. Díaz-Levicoy et al Derechos Reservados (C) 2017 Revista digital Matemática, Educación e Internet (http://tecdigital.tec.ac.cr/revistamatematica/) 
barras o un gráfico de sectores, en este último caso se usa la noción de área. Además, en la construcción de un gráfico estadístico se debe considerar la naturaleza de los datos, pues no todos los gráficos son adecuados para su representación (Díaz-Levicoy, 2014[12]), errores que ya han sido observados por autores como Li y Shen (1992[24]).

En lo que sigue describimos algunos estudios en los que se da cuenta de los errores cometidos por estudiantes de Educación Primaria.

Guimarães, Gitirana y Roazzi (2001[22]) reportan los resultados de dos actividades que se relacionan con la construcción de gráficos de barras por 107 estudiantes de $3^{\circ}$ de Educación Primaria en Pernambuco (Brasil). Los resultados muestran que la construcción de gráficos para datos nominales (47,7\%) es más sencillo que la de datos ordinales ( $25 \%$ aprox.), aunque dichos resultados pueden verse influidos por el ámbito numérico que se trabaja, así como por la escala que deben usar, que son diferentes en cada actividad. Sobre este último punto (las escalas) se observan su ausencia y dificultades para construirlas en forma adecuada, aunque la ausencia se podría justificar a que no se percibe su utilidad, por tener una visión del gráfico de barras como si fuese un pictograma.

Fernandes, Morais y Lacaz (2011[16]) muestran los resultados de una investigación sobre el desempeño, dificultades y errores en la construcción de gráficos estadísticos de108 estudiantes de $9^{\circ}$ año de Educación Fundamental en Braga (Portugal). Para recoger la información se pidió a los estudiantes que desarrollaran dos actividades de construcción del gráfico más apropiado de acuerdo a la naturaleza de los datos -en la primera se presentan las edades y sexo de 28 estudiantes, de esta información se pide dos tareas: construir un gráfico que represente las edades (cuantitativa discreta) y otro que represente las edades según sexo (comparación de cuantitativas discretas según una cualitativa); la segunda está basada en la construcción de un gráfico con los años de vida de 21 animales (cuantitativa continua)-. Entre los resultados se destacan: la dificultad de los estudiantes para construir gráficos estadísticos; los estudiantes presentaron mejor desempeño en la representación de una variable cuantitativa discreta $(61 \%)$, seguida de la representación para comparar un variable cuantitativa discreta de acuerdo a una cualitativa(35\%), y con un rendimiento muy bajo la de representar una variable cuantitativa continua $(2 \%)$. Los errores observados están asociados a la elección de un gráfico inadecuado, ausencia de títulos y etiquetas de los ejes, escalas inadecuadas y falta de rigor al construir.

Walichinski y Santos Junior (2013[31]) describen los resultados que se han obtenido en una secuencia didáctica para el trabajo con gráficos y tablas estadísticas con 22 estudiantes de $7^{\circ}$ grado de Educación Primaria en Ponta Grossa (Brasil), los que tenían una edad media de 12 años. Para el desarrollo de esta secuencia didáctica se usaron tres momentos: pre-test, intervención didáctica y pos-test. Los resultados de la experiencia muestran que los niños despertaron interés por participar de las clases, mejoraron la disposición a trabajar las actividades que se planteaban, y generaban una mayor interacción entre el profesor y los niños. Además, se observó un avance significativo en relación a la adquisición de conocimiento, es decir, se logró que los niños percibieran la necesidad de colocar título, categoría a los ejes o la fuente de los datos al construir gráficos estadísticos.

Evangelista, Oliveira y Ribeiro (2014[14]) describe los resultados de una investigación que analizaba el desempeño de 46 estudiantes de $5^{\circ}$ año de una escuela pública de Olinda (Brasil). A los estudiantes se les pidió construir dos gráficos estadísticos, sin especificar su tipo. Los resultados indican que el $88,1 \%$ de los estudiantes fue capaz de construir los dos gráficos; ningún estudiante colocó título a los gráficos construidos; solo el 3,3\% del total de los estudiantes fue capaz de asignar nombre a los ejes. 
También, la mayoría de los estudiantes $(77,2 \%)$ consiguió describir las variables del eje $\mathrm{X}$, y solo el 19,6\% consiguió establecer adecuadamente la escala; situación que confirma lo expresado por (Silva, 2014 ), al expresar las dificultades que tienen los estudiantes al trabajar con escalas no unitarias. En general, los estudiantes son capaces de realizar los gráficos pedidos, pero pocos son capaces de asignar correctamente títulos, nombre de los ejes y escalas.

Ruiz (2015[29]) realiza un estudio con 31 escolares de quinto grado de Educación Primaria en un colegio de Bogotá (Colombia), para indagar sobre los errores y dificultades que presentan ante una actividad de construcción de gráficos. En las producciones de los estudiantes se observan los siguientes errores y dificultades:

- Usar un gráfico no adecuado a la naturaleza de los datos representados.

- Dificultades en el manejo de las escalas en la presentación de los datos. Esto es, ejes que presentan divisiones no proporcionales, que la escala es inadecuada.

- Problemas de simetría o paralelismo entre figuras.

- Ausencia de rótulos que permite una mejor comprensión de la información mostrada en el gráfico.

Cruz (2013[8]), en su estudio con estudiantes de tercero de primaria, mencionado anteriormente, observa los siguientes errores asociados a la construcción de un gráfico de barras:

- Falta de leyendas en los ejes horizontales y verticales

- Barras con diferente ancho y con separación no uniforme entre ellas.

- No colocar correctamente las categorías de las variables mostradas en el gráfico.

- No seleccionar una unidad constate para definir la escala y no indicarlas en los respectivos ejes.

\subsection{Conclusión}

Los estudios anteriores relacionados con la lectura de gráficos estadísticos dejan en evidencia dificultades que conlleva para los estudiantes. Del mismo modo, las investigaciones sobre construcción de gráficos muestran que los estudiantes de Educación Primaria presentan errores, tales como: elección inadecuada del gráfico; falta de proporcionalidad en sus elementos (barras con alturas no proporcionales

y separación no uniforme de las mismas, iconos no proporcionales en los pictogramas, etc.); dificultad para identificar los ejes, ausencia de leyendas y rótulos.

Algunas de estas dificultades se pueden atribuir a que estos temas no se trabajan en forma adecuada y rigurosa, pues diversas investigaciones muestran los problemas que presentan los mismos profesores, en formación o en activo, al trabajar con estas representaciones (e.g., Arteaga, Batanero, Contreras y

Investigaciones sobre gráficos estadísticos en Educación Primaria: revisión de la literatura. D. Díaz-Levicoy et al Derechos Reservados (C) 2017 Revista digital Matemática, Educación e Internet (http://tecdigital.tec.ac.cr/revistamatematica/) 
Cañadas, 2016[3]; González, Espinel y Ainley, 2011[20]; Rodríguez y Sandoval, 2012[28]) o, como mencionan Wall y Benson (2009), porque los profesores no se siente cómodos enseñando estos temas.

Esta revisión de la literatura puede aportar elementos teóricos y antecedentes para el desarrollo de estudios en aquellos países que la investigación sobre gráficos estadísticos es más escasa (ejemplo, Chile, España, México, entre otros), teniendo en cuenta que los temas de estadística y probabilidad se han incluido recientemente en las directrices curriculares de Educación Primaria.

\section{Bibliografía}

[1] A. Alaminos, Gráficos. Cuadernos metodológicos, Madrid, Centro de Investigaciones Sociológicas, 1993.

[2] P. Arteaga, Evaluación de conocimientos sobre gráficos estadísticos y conocimientos didácticos de futuros profesores [Tesis Doctoral], Granada, Universidad de Granada, 2011.

[3] P. Arteaga, C. Batanero, J.M. Contreras; G. Cañadas.. Evaluación de errores en la construcción de gráficos estadísticos elementales por futuros profesores. Revista Latinoamericana de Investigación en Matemática Educativa, 2016, 19(1), p. 15-40.

[4] C. Batanero; P. Arteaga; B. Ruiz, Análisis de la complejidad semiótica de los gráficos producidos por futuros profesores de educación primaria en una tarea de comparación de dos variables estadísticas, Enseñanza de las Ciencias, 2010, 28(1), p. 141?154.

[5] C. Batanero; C. Díaz; J.M. Contreras; R. Roa, El sentido estadístico y su desarrollo, Números, 2013, 83, p. $7-18$.

[6] Bertin, J. Semiologie graphique, Paris, Gauthier-Villars, 1967.

[7] L.M.T.L. Carvalho; T.M.D.M. Campos; C.E.F. Monteiro, Aspectos visuais e conceituais nas interpretações de gráficos de linhas por estudantes, Boletim de Educação Matemática, 2011, 24(40), p. 679-700.

[8] A. Cruz, Erros e dificuldades de alunos de $1^{\circ}$ ciclo na representação de dados estatísticos [Tesis de Máster], Lisboa, Universidade de Lisboa, 2013.

[9] F. R. Curcio (1987). Comprehension of mathematical relationships expressed in graphs. Journal for Research in Mathematics Education, 1987, 18(5), p. 382-393.

[10] F. R. Curcio, Developing graph comprehension, Reston, NCTM, 1989.

[11] G. Del Pino; S. Estrella, Educación estadística: Relaciones con la matemática, Pensamiento Educativo. Revista de Investigación Educacional Latinoamericana, 2012, 49(1), p. 53-64.

[12] D. Díaz-Levicoy, Un estudio empírico de los gráficos estadísticos en libros de texto de Educación Primaria española [Trabajo Fin de Máster], Granada, Universidad de Granada, 2014. 
[13] B. Evangelista, Aprendendo a representar escalas em gráficos: um estudo de intervenção [Tesis de Mestrado], Recife, Universidade Federal de Pernambuco, 2014.

[14] B. Evangelista; F.S.M.A. Oliveira; P.M. Ribeiro, Analizando a construção de gráficos de alunos do $5^{\circ}$ ano do ensino fundamental. Congresso Nacional de Educação (CONEDU), Campina Grande, Brasil. 2014.

[15] J.A. Fernandes; P.C. Morais, Leitura e interpretação de gráficos estatísticos por alunos do $9^{\circ}$ ano de escolaridade, Educação Matemática Pesquisa, 2011, 13(1), p. 95-115.

[16] J.A. Fernandes; P.C. Morais; T.V.S. Lacaz (2011). Representação de dados através de gráficos estatísticos por alunos do $9^{\circ}$ ano de escolaridade. Anais da XIII Conferência Interamericana de Educação Matemática, Recife, Brasil. 2011.

[17] S. Friel; F. Curcio; G. Bright, Making sense of graphs: critical factors influencing comprehension and instructional implications. Journal for Research in Mathematics Education, 2001, 32(2), p. 124-158.

[18] I. Gal, Adult's statistical literacy: Meaning, components, responsibilities, International Statistical Review, 2002, 70(1), p. 1-25.

[19] N. Glazer, Challenges with graph interpretation: a review of the literatura, Studies in Science Education, 2001, 47(2), p. 183-210.

[20] M.T. González; M.C. Espinel; J. Ainley, Teachers? graphical competence, en C. Batanero, G. Burrill y C. Reading, C (eds.), Teaching statistics in school mathematics-Challenges for teaching and teacher education, New York, Springer, 2011, p. 187-197.

[21] G.L. Guimarães, Interpretando e construindo gráficos de barras [Tesis doctoral], Pernambuco, Universidade Federal de Pernambuco, 2002.

[22] G.L. Guimarães; V. Gitirana; A. Roazzi, Interpretando e construindo gráficos. 24 Reunión Anual de ANPeD. Caxambu, Brasil. 2001.

[23] S. M. Kosslyn, Graphics and human information processing. Journal of the American Statistical Association, 1985, 80(391), p. 499-512.

[24] D. Y. Li; S. M. Shen, Students?weaknesses in statistical projects, Teaching Statistics, 1992, 14(1), p. 2-8.

[25] MINEDUC, Matemática educación básica. Bases curriculares, Santiago, Unidad de Currículum y Evaluación, 2012.

[26] MECD, Real Decreto 126/2014, de 28 de febrero, por el que se establece el currículo básico de la Educación Primaria, Madrid, 2014.

[27] A. Pagan; A.P. Leite; S. Magina; I. Cazorla, A leitura e interpretação de gráficos e tabelas no Ensino Fundamental e Médio. $2^{\circ}$ Simpósio Internacional de Pesquisa em Educação Matemática (SIPEMAT). 
Recife, Brasil. 2008.

[28] F. Rodríguez; P.R. Sandoval, Habilidades de codificación y descodificación de tablas y gráficos estadísticos: un estudio comparativo en profesores y alumnos de pedagogía en Enseñanza Básica, Avaliação: Revista da Avaliação da Educação Superior, 2012; 17(1), p. 207-235.

[29] A. Ruiz, Un estudio de caso sobre errores y dificultades observadas en la elaboración de algunas gráficas estadísticas, Revista Góndola. Enseñanza y Aprendizaje de las Ciencias, 2015, 10(1), p. 26-39.

[30] H. Wainer, Understanding graphs and tables, Educational Researcher, 1992, 21(1), p. 14-23.

[31] D. Walichinski; G. Santos Junior, Contribuições de uma sequência de ensino para o processo de ensino e aprendizagem de gráficos e tabelas segundo pressupostos da contextualização, Revista Iberoamericana de Educación Matemática, 2013, 35, p. 19-42.

[32] Y. Wu, Singapore secondary school students'understanding of statistical graphs. 10th International Congress on Mathematics Education. Copenhagen, Dinamarca. 2004. 